\title{
Speed of convergence in first passage percolation and geodesicity of the average distance
}

\author{
Romain Tessera \\ Laboratoire de Mathématiques, Bâtiment 425, Université Paris-Sud 11, 91405 Orsay, France. E-mail: romain.tessera@math.u-psud.fr
}

Received 30 June 2015; revised 19 November 2016; accepted 27 November 2016

\begin{abstract}
We give an elementary proof that Talagrand's sub-Gaussian concentration inequality implies a limit shape theorem for first passage percolation on any Cayley graph of $\mathbb{Z}^{d}$, with a speed of convergence $\lesssim\left(\frac{\log n}{n}\right)^{1 / 2}$. Our approach, which does not use the subadditive theorem, is based on proving that the average distance $\mathbb{E} d_{\omega}$ on $\mathbb{Z}^{d}$ is close to being geodesic. Our key observation, of independent interest, is that the problem of estimating the rate of convergence for the average distance is equivalent (in a precise sense) to estimating its "level of geodesicity".
\end{abstract}

Résumé. Nous démontrons de manière élémentaire que l'inégalité de concentration sous-Gaussienne de Talagrand implique le théorème de forme limite en percolation de premier passage sur un graphe de Cayley quelconque de $\mathbb{Z}^{d}$, avec un terme d'erreur $\lesssim\left(\frac{\log n}{n}\right)^{1 / 2}$. Au lieu de nous baser sur le théorème sous-additif comme dans les approches classiques, notre preuve repose sur le fait de montrer que la distance moyenne est en un sens quantitatif, presque géodésique. Notre observation centrale, qui présente un intérêt indépendant, est que le problème d'estimer la vitesse de convergence de la distance moyenne vers la norme limite est équivalent (en un sens précis) à celui d'estimer son «niveau de géodécisité. »

MSC: 46B85; 20F69; 22D10; 20E22

Keywords: First passage percolation; Limit shape theorem; Speed of convergence

\section{Introduction}

First passage percolation (FPP) is a way to randomly perturb the distance on a connected graph. Let us recall how this random process is defined.

Consider a connected non-oriented graph $X$, whose set of vertices (resp. edges) is denoted by $V$ (resp. $E$ ). We first define the notion of weighted graph metric on $V$. For every function $\omega: E \rightarrow(0, \infty)$, we equip $V$ with the weighted graph metric $d_{\omega}$, where each edge $e$ has weight $\omega(e)$. In other words, for every $x, y \in V, d_{\omega}(x, y)$ is defined as the infimum over all path $p=\left(e_{1}, \ldots, e_{m}\right)$ joining $x$ to $y$ of $\ell_{f}(p):=\sum_{i=1}^{m} \omega\left(e_{i}\right)$. Denote by $d$ the graph metric on $V$, corresponding to the constant function $\omega=1$.

Let $v$ be a probability measure supported on $[0, \infty)$. The random metric of first passage percolation consists in choosing the weight $\omega(e)$ independently according to $v$. Note that $\mathbb{E} d_{\omega}(x, y)$ defines a distance on $V$, that we call the average distance and denote by $\bar{d}(x, y)$.

A central result in FPP is the following Gaussian concentration inequality due to Talagrand.

Theorem ([13, Proposition 8.3]). Suppose that $\omega(e)$ has an exponential moment: i.e. there exists $c>0$ such that $\mathbb{E} \exp (c \omega(e))<\infty$. Then there exists $C_{1}$ and $C_{2}$ such that for every graph $X=(V, E)$, for every pair of vertices $x, y$, 
and for every $u \geq 0$,

$$
P\left(\left|d_{\omega}(x, y)-\bar{d}(x, y)\right| \geq u\right) \leq C_{1} \exp \left(-C_{2} \min \left\{\frac{u^{2}}{d(x, y)}, u\right\}\right) .
$$

\subsection{A quantitative limit shape theorem for Cayley graphs of $\mathbb{Z}^{d}$}

\section{Basic assumptions}

In order to avoid useless repetitions, let us once and for all list the technical assumptions on the edge's length distribution $v$, that will be required in most of our statements.

$\left(A_{1}\right)$ We assume that $v$ has an exponential moment, and therefore satisfies (1.1) for some constants $C_{1}$ and $C_{2}$ (this assumption can probably be relaxed but we choose not to focus on this aspect here).

$\left(A_{2}\right)$ We also suppose that there exists $a>0$ such that $\bar{d}(x, y) \geq a d(x, y)$ for all $x, y \in V$.

Recall that given a group $G$ and a generating subset $S \subset G$, the Cayley graph $(G, S)$ is the graph whose vertices are elements of $G$ and whose edges are pairs of elements $g, h \in G$ such that $g=h s^{ \pm 1}$ for some $s \in S$. Note that the $G$ action on itself by left-translations induces an action on $(G, S)$ by automorphisms which is transitive on the set of vertices. The standard Cayley graph of $\mathbb{Z}^{d}$, sometimes called the euclidean grid is $\left(\mathbb{Z}^{d}, S\right)$, where $S$ is the standard basis of $\mathbb{Z}^{d}$.

When one works with the standard Cayley graph of $\mathbb{Z}^{d}$, the second assumption is satisfied exactly when $v(\{0\})<p_{c}$, where $p_{c}$ is the critical probability of percolation on $\mathbb{Z}^{d}$ [9]. For more general graphs, we show that the second condition is fulfilled provided that $v(\{0\})<1 / k$, where $k$ is an upper bound on the degree of the graph (see Corollary A.2).

Observe that $\bar{d} \leq(\mathbb{E} \omega(e)) d$. In the sequel we denote $b:=\mathbb{E} \omega(e)$. It follows that under our second assumption, $d$ and $\bar{d}$ are actually bi-Lipschitz equivalent, more precisely,

$$
a d \leq \bar{d} \leq b d .
$$

We shall adopt the following notation: given $v \in V$ and $r>0$, let $\bar{B}(v, r)$ (resp. $B_{\omega}(v, r)$ ) denote the ball of radius $r$ for the average distance $\bar{d}$ (resp. for the random distance $d_{\omega}$ ).

We give a new proof of the following theorem, which in the case of the euclidean grid in $\mathbb{Z}^{d}$ can be attributed to Alexander (see the discussion below).

Theorem 1.1 (Quantitative asymptotical shape theorem). We consider a Cayley graph of $\mathbb{Z}^{d}$, associated to some finite generating subset. We assume $\left(A_{1}\right)$ and $\left(A_{2}\right)$ are satisfied. There exists a norm $\|\cdot\|$ on $\mathbb{R}^{d}$ such that for a.e. $\omega$, there exists $C>0$ and $n_{0}$ such that for all $n \geq n_{0}$,

$$
B_{\|\cdot\|}\left(0, n-C(n \log n)^{1 / 2}\right) \cap \mathbb{Z}^{d} \subset B_{\omega}(0, n) \subset B_{\|\cdot\|}\left(0, n+C(n \log n)^{1 / 2}\right) .
$$

The fact that the rescaled ball converges to a convex body was first proved by Kesten [9], extending previous work by Richardson [12] and Cox-Durrett [5] (for background see [1,8,9]). The first quantitative estimates, given by Kesten [10], depended on the dimension. These estimates were later improved by Alexander [2] who proved an error term in $O\left(n^{1 / 2} \log n\right)$.

More recently, a Gaussian estimate for the lower tail has recently been obtained under a quadratic moment condition in [6] (see also $[7,15,16])$. Following the strategy of proof of [2] (itself inspired from [10]) they manage to deduce the right-hand side inequality of (1.3) under this low moment condition.

Actually, Alexander's bounds rely on a sub-exponential concentration inequality due to Kesten. If one replaces the latter by Talagrand's sub-Gaussian's concentration inequality (1.1), his proof yields (1.3).

Finally let us mention that Theorem 1.1 is likely to remain far from optimal. Indeed, we recall that physicists believe that the error term for $\mathbb{Z}^{2}$ should be $n^{1 / 3}$. However, it is not clear what rate should be expected in higher dimensions (see [10] for a more detailed account and the relevant references).

The proof of Theorem 1.1 is quite different from Alexander's approach. On the one hand, it breaks down the main result into two independent statements that we shall describe below: one is a straightforward bound on the fluctuations 
about the average distance, while the other one bounds the speed of convergence of the rescaled average distance to the limit norm. This last step can also be decomposed into two independent results: an easy one which is based on $\left(A_{1}\right)$ and is valid for any graph with polynomial growth, and a more subtle, purely geometric statement that explicitly uses the abelian group structure of $\mathbb{Z}^{d}$.

Another interesting feature of this new approach is the fact that it does not use the subadditive ergodic theorem, unlike the previous ones. In [3], we exploit this to obtain a limit shape theorem for any Cayley graphs of polynomial growth, which did not seem to be approachable by previous methods.

\subsection{Fluctuations around the average metric / speed of convergence for the average metric}

We now describe the two main estimates that are needed in our proof of Theorem 1.1. Let us start with the following straightforward consequence of Talagrand's concentration inequality.

Proposition 1.2 (Fluctuations about the average distance). Let $d>0$ and $K>0$. Then there exists $C>0$ only depending on $d$ and $K$ such that the following holds. Let $X=(V, E)$ be a graph. We assume $\left(A_{1}\right)$ and $\left(A_{2}\right)$ are satisfied. Let $r_{n} \in \mathbb{N}$ be an increasing sequence, and let $o_{n}$ be a sequence of vertices such that $\left|B\left(o_{n}, r_{n}\right)\right| \leq K r_{n}^{d}$. Then for a.e. $\omega$, there exists $n_{0}$ such that for $n \geq n_{0}$,

$$
\sup _{x, y \in B\left(o_{n}, r_{n}\right)}\left|d_{\omega}(x, y)-\bar{d}(x, y)\right| \leq C\left(r_{n} \log r_{n}\right)^{1 / 2} .
$$

We deduce from the previous proposition that there exists $C^{\prime}$ such that for a.e. $\omega$ there exists $n_{0}$ such that for all $n \geq n_{0}$, one has

$$
\bar{B}\left(0, r_{n}-C^{\prime}\left(r_{n} \log r_{n}\right)^{1 / 2}\right) \subset B_{\omega}\left(0, r_{n}\right) \subset \bar{B}\left(0, r_{n}+C^{\prime}\left(r_{n} \log r_{n}\right)^{1 / 2}\right) .
$$

The complementary (and main) step in the proof of Theorem 1.1 therefore consists in estimating the speed of convergence of the rescaled ball for the average distance on $\mathbb{Z}^{d}$.

Theorem 1.3 (Asymptotical shape theorem for the average distance). We consider a Cayley graph of $\mathbb{Z}^{d}$. We assume $\left(A_{1}\right)$ and $\left(A_{2}\right)$ are satisfied. There exist a norm $\|\cdot\|$ on $\mathbb{R}^{d}, C>0$ and $n_{0}$ such that for all $n \geq n_{0}$,

$$
B_{\|\cdot\|}\left(0, n-C(n \log n)^{1 / 2}\right) \cap \mathbb{Z}^{d} \subset \bar{B}(0, n) \subset B_{\|\cdot\|}\left(0, n+C(n \log n)^{1 / 2}\right) .
$$

\subsection{On how to quantify being geodesic "in an asymptotical way"}

In order to explain the strategy behind the proof of Theorem 1.3, we need to introduce the notion of strong asymptotical geodesicity. Before giving a formal definition, let us review two important properties of a (discrete) geodesic metric space. Recall that given a connected graph $(V, E)$, the vertex set $V$ equipped with graph distance satisfies the following two (equivalent) properties:

- for any $x, y \in V$, such that $d(x, y)=n$, there exists a sequence of vertices $x=x_{0}, x_{1}, \ldots, x_{n}=y$ such that $d\left(x_{i}, x_{i+1}\right)=1$ for all $0 \leq i<n$;

- for every $n<n^{\prime}$ and every $x \in V$, the distance from any vertex in $B\left(x, n^{\prime}\right)$ to $B(x, n)$ is at most $n^{\prime}-n$. This can also be formulated as

$$
B\left(x, n^{\prime}\right) \subset[B(x, n)]_{n^{\prime}-n},
$$

where $[A]_{t}$ denotes the $t$-neighborhood of the subset $A$.

This suggests at least two ways of defining being geodesic "in an asymptotical way":

The first one is called "inner metric" in [11], or "asymptotical geodesic metric" [4]. A metric space $X$ is called asymptotically geodesic if for all $\varepsilon>0$ there exists $\alpha$ such that for all $x, y \in X$, there exists a sequence $x=x_{0}, \ldots, x_{m}=y$ such that $d\left(x_{i}, x_{i+1}\right) \leq \alpha$, and $\sum_{i=0}^{m-1} d\left(x_{i}, x_{i+1}\right) \leq(1+\varepsilon) d(x, y)$. 
The second one is "monotone geodesic metric" as defined by the author in [14]. Monotone geodesicity is defined by requiring the existence of a constant $T$ such that for all $x$ and all $r, B(x, r+1) \subset[B(x, r)]_{T}$. One can relax this assumption by letting $T$ depend on $r$.

Asymptotical geodesicity was used by Pansu to obtain a limit shape theorem for Cayley graphs of nilpotent groups [11], while monotone geodesicity was used to bound the size of the spheres in graphs with the Doubling Property. ${ }^{1}$ In some sense both notions have to do with controlling the error terms when estimating the size of large balls. It is therefore not surprising that the notion that we need here is a quantitative combination of these two.

Definition 1.4 (Strongly Asymptotically Geodesic spaces). Let $N: \mathbb{R}_{+} \rightarrow \mathbb{R}_{+}$be an increasing function such that $\lim _{\alpha \rightarrow \infty} N(\alpha)=\infty$. A metric space $X$ is called $\operatorname{SAG}(N)$ if there exists $\alpha_{0} \geq 0$ such that for all integer $m \geq 1$, and for all $x, y \in X$ such that $d(x, y) / m \geq \alpha_{0}$, there exists a sequence $x=x_{0} \ldots, x_{m}=y$ satisfying, for all $0 \leq i \leq m-1$,

$$
d\left(x_{i}, x_{i+1}\right) \leq \alpha\left(1+\frac{1}{N(\alpha)}\right)
$$

where $\alpha=d(x, y) / m$; and for all large enough $r$,

$$
B\left(x,\left(1+\frac{1}{N(r)}\right) r\right) \subset[B(x, r)]_{\frac{6 r}{N(r)}} .
$$

It turns out that the SAG property can be deduced (for a large class of functions $N$ ) from the following much simpler property (suggested to me by Xuan Wang).

Definition 1.5. Given a increasing function $N: \mathbb{R}_{+} \rightarrow \mathbb{R}_{+}$such that $\lim _{\alpha \rightarrow \infty} N(\alpha)=\infty$, we say a metric space $X$ is $\mathrm{SAG}^{*}(N)$ if there exists $\alpha_{0}>0$ such that the following holds: for all $x, y \in X$ satisfying $d(x, y)=r \geq \alpha_{0}$, and $\lambda \in[0,1]$, we can find $z \in X$ such that

$$
\left(\lambda-\frac{1}{N(r)}\right) d(x, y) \leq d(x, z) \leq\left(\lambda+\frac{1}{N(r)}\right) d(x, y),
$$

and

$$
\left(1-\lambda-\frac{1}{N(r)}\right) d(x, y) \leq d(y, z) \leq\left(1-\lambda+\frac{1}{N(r)}\right) d(x, y) .
$$

The connection between these two notions is given by the following proposition.

Proposition 1.6 (SAG* implies SAG). Let $\varepsilon, \delta>0$ and consider an increasing function $N:(0, \infty) \rightarrow(0, \infty)$ such that:

- for all large enough $x$ and $t \geq 1 / 2$,

$$
N(x t) \geq \delta N(x) t^{\varepsilon},
$$

- for large enough $x$, the function $x \rightarrow x\left(1+\frac{1}{N(x)}\right)$ is non decreasing.

If a metric space $X$ is $\mathrm{SAG}^{*}(N)$, then it is $\mathrm{SAG}(c N)$ for some $c>0$.

Note that the above condition (for $t=1 / 2$ ) implies that there is a constant $K$ such that that $N(2 y) \leq K N(y)$ for all large enough $y$. Observe that any function of the form $N(\alpha)=\alpha^{u}(\log \alpha)^{v}$ with $u>0$ and $v \in \mathbb{R}$ satisfies the condition of the proposition.

\footnotetext{
${ }^{1}$ A graph satisfies the Doubling Property if there exists a constant $C>0$ such that for all $x \in V$ and all $r>0,|B(x, 2 r)| \leq C|B(x, r)|$.
} 


\subsection{Strong asymptotic geodesicity of the average metric}

The proof of Theorem 1.3 relies on the following result, of independent interest. Observe that the only geometric property of the graph that is required is some sub-polynomial volume growth condition. It applies for instance to first passage percolation on fractal graphs, Cayley graphs of nilpotent groups (see [3]), or random environments such as the infinite cluster of supercritical percolation on $\mathbb{Z}^{d}$.

Proposition 1.7 (The average metric is $\left.\operatorname{SAG}^{*}\left((\alpha / \log \alpha)^{1 / 2}\right)\right)$. Let $X=(V, E)$ be a graph. Suppose that there exists $K>0$ and $d>0$ such that for all $o \in V$ and all $r>0,|B(o, r)| \leq K r^{d}$. We assume $\left(A_{1}\right)$ and $\left(A_{2}\right)$ are satisfied. Then there exists a constant $c>0$ such that the metric $\bar{d}$ is $\operatorname{SAG}^{*}(N)$ with

$$
N(\alpha)=c\left(\frac{\alpha}{\log \alpha}\right)^{1 / 2}
$$

In particular it is $\operatorname{SAG}\left(c^{\prime} N\right)$ for some $c^{\prime}>0$.

The idea behind the proof of Proposition 1.7 is relatively simple: it consists in exploiting the concentration inequality due to Talagrand to show that $\bar{d}$ being close to $d_{\omega}$ with large probability, since $d_{\omega}$ is geodesic, then $\bar{d}$ cannot be too far from being geodesic.

Let us be more precise about what we mean by " $d_{\omega}$ is geodesic": by definition, for all $x, y \in V$ and all $\varepsilon>0$, there exists a simple path $p=\left(e_{1}, \ldots, e_{k}\right)$ in $X$ joining $x$ to $y$, and such that $\sum_{i} \omega\left(e_{i}\right) \leq d_{\omega}(x, y) \leq \sum_{i} \omega\left(e_{i}\right)+\varepsilon$. If $\varepsilon=0$, then such a path is called an $\omega$-geodesic between $x$ and $y$. Although this is not used in this paper, one can easily deduce from Proposition 1.2 that the polynomial growth assumption is enough to ensure that every pair of points is almost surely joined by some $\omega$-geodesic (see Claim 3.2).

\subsection{A geometric statement about invariant metrics on $\mathbb{Z}^{d}$}

So far we have not used any specific feature of $\mathbb{Z}^{d}$. It turns out that the connection between strong asymptotical geodesicity of the average metric and the limit shape theorem follows from a very general result about invariant metrics on $\mathbb{Z}^{d}$.

In the sequel, an increasing function $\phi: \mathbb{R}_{+} \rightarrow \mathbb{R}_{+}$is called sublinearly doubling if there exists a function $\eta:$ $\mathbb{R}_{+} \rightarrow \mathbb{R}_{+}$satisfying $\lim _{\lambda \rightarrow \infty} \eta(\lambda) / \lambda=0$ such that for all $\lambda>0$ and $r>0, \phi(\lambda r) \leq \eta(\lambda) \phi(r)$. A distance $d$ on a group $G$ is called left-invariant if $d(g h, g k)=d(h, k)$ for all $g, h, k \in G$. If the group is abelian, then we simply say that the distance is invariant.

Proposition 1.8 (Strong Asymptotical Geodesicity versus Limit Shape). We let $\delta$ be some invariant metric on $\mathbb{Z}^{d}$. We let $\phi: \mathbb{R}_{+} \rightarrow[1,+\infty)$ be an increasing, sublinearly doubling function. The following two assertions are equivalent.

(i) There exists a constant $c>0$ such that $\delta$ is $\operatorname{SAG}(N)$ with

$$
N(\alpha) \geq c \phi(\alpha),
$$

for $\alpha$ large enough.

(ii) There exists a norm $\|\cdot\|$ on $\mathbb{R}^{d}$ and $C>0$ such that for all large enough $n$,

$$
B_{\|\cdot\|}\left(0, n-\frac{C n}{\phi(n)}\right) \cap \mathbb{Z}^{d} \subset B_{\delta}(0, n) \subset B_{\|\cdot\|}\left(0, n+\frac{C n}{\phi(n)}\right) .
$$

It is now clear that Theorem 1.3 results from Proposition 1.8 (for $\delta=\bar{d}$, and $\phi(\alpha)=(\alpha / \log \alpha)^{1 / 2}$ ) and Proposition 1.7 .

We hope that Proposition 1.8 will be useful for future attempts to improve the known estimates -both from above and from below - on the speed of convergence in Theorem 1.3. 


\section{Organization}

In Section 2 we provide the quick proof of Proposition 1.2. Section 3 is dedicated to the proof of Proposition 1.7. These first two short sections are the only ones concerned with probabilistic arguments (recall that these statements are proved for general graphs with a sub-polynomial growth condition). Section 4 is dedicated to the proof of Proposition 1.6, which holds for any metric space. In the last two sections (Section 5 and Section 6) we prove both implications of Proposition 1.8, which is a statement about invariant metrics on $\mathbb{Z}^{d}$. The different sections can be read independently.

\section{Proof of Proposition 1.2}

Let $D$ be a constant to be determined later. Applying Talagrand's theorem, we obtain that for all large enough $r$, and all $x, y$ such that $d(x, y) \leq r$,

$$
P\left(\left|d_{\omega}(x, y)-\bar{d}(x, y)\right|^{2} \geq \operatorname{Dr} \log r\right) \leq C_{1} \exp \left(-C_{2} D \log r\right) .
$$

Now, letting $D=(2 d+2) / C_{2}$, we deduce that for all large enough $r$, and all $x, y$ such that $d(x, y) \leq r$,

$$
P\left(\left|d_{\omega}(x, y)-\bar{d}(x, y)\right|^{2} \geq \operatorname{Dr} \log r\right) \leq C_{1} r^{-2 d-2} .
$$

Hence for $n$ large enough,

$$
P\left(\sup _{x, y \in B\left(o_{n}, r_{n}\right)}\left|d_{\omega}(x, y)-\bar{d}(x, y)\right|^{2} \geq D r_{n} \log r_{n}\right) \leq C_{1} r_{n}^{-2 d-2}\left|B\left(o_{n}, r_{n}\right)\right|^{2} \leq C_{1} K r_{n}^{-2} \leq C_{1} K n^{-2},
$$

the last inequality following from the fact that $r_{n}$ is an increasing sequence of non-negative integers. Proposition 1.2 now follows from the fact that $\sum_{n} n^{-2}<\infty$ (with $C=D$ ).

\section{The average metric is SAG}

The goal of this section is to prove Proposition 1.7, which immediately follows from the following proposition.

Proposition 3.1. Let $d>0$ and $K>0$, and let $r_{n} \in \mathbb{N}$ be an increasing sequence. Then there exists $c>0$ and $n_{0}$ such that the following holds. Let $X=(V, E)$ be a graph. We assume $\left(A_{1}\right)$ and $\left(A_{2}\right)$ are satisfied, and that there exists a sequence of vertices $o_{n}$ such that $\left|B\left(o_{n}, r_{n}\right)\right| \leq K r_{n}^{d}$. Then, for $n \geq n_{0}$, for all $x, y \in B\left(o_{n}, a r_{n} /(32 b)\right)$ and for all $0 \leq \lambda \leq 1$, there exists a vertex $z \in B\left(o_{n}, r_{n}\right)$ such that

$$
|\lambda \bar{d}(x, y)-\bar{d}(x, z)| \leq \frac{r_{n}}{N\left(r_{n}\right)},
$$

and

$$
|(1-\lambda) \bar{d}(x, y)-\bar{d}(z, y)| \leq \frac{r_{n}}{N\left(r_{n}\right)},
$$

where

$$
N(r)=c\left(\frac{r}{\log r}\right)^{1 / 2} .
$$

Proof. In the proof of Proposition 1.2, we established that

$$
P\left(\sup _{x, y \in B\left(o_{n}, r_{n}\right)}\left|d_{\omega}(x, y)-\bar{d}(x, y)\right|^{2} \geq D r_{n} \log r_{n}\right) \leq C_{1} K r_{n}^{-2},
$$


with $D=(2 d+2) /\left(C_{2} b^{2}\right)$ (remember that $C_{1}$ and $C_{2}$ are the constants appearing in the conclusion of Talagrand's theorem). Let $n_{0}$ be the smallest integer so that $C_{1} K r_{n_{0}}^{-2}<1$. Then for all $n \geq n_{0}$, there exists $\omega$ (depending on $n$ ) such that

$$
\sup _{z_{1}, z_{2} \in B\left(o, r_{n}\right)}\left|d_{\omega}\left(z_{1}, z_{2}\right)-\bar{d}\left(z_{1}, z_{2}\right)\right| \leq D\left(r_{n} \log r_{n}\right)^{1 / 2} .
$$

Assume in addition that $n_{0}$ is large enough so that $D\left(r_{n} \log r_{n}\right)^{1 / 2} \leq a r_{n} / 16$ for all $n \geq n_{0}$.

Claim 3.2. Let $\gamma$ be some path between $x$ and $y$ whose $\omega$-length is $\leq d_{\omega}(x, y)+a r_{n} / 16$. Then $\gamma$ is contained in the ball $B\left(o_{n}, r_{n}\right)$ (in particular there exists an $\omega$-geodesic between $x$ and $y$ ).

Proof. Indeed, suppose there is $1 \leq i \leq k$ such that $d\left(o_{n}, \gamma(i)\right)=r_{n}$, then by triangular inequality,

$$
d(x, \gamma(i)) \geq d\left(o_{n}, \gamma(i)\right)-d\left(o_{n}, x\right) \geq r_{n} / 2,
$$

hence $\bar{d}(x, \gamma(i)) \geq a r_{n} / 2$. So (3.1) implies that

$$
d_{\omega}(x, \gamma(i)) \geq \bar{d}(x, \gamma(i))-D\left(r_{n} \log r_{n}\right)^{1 / 2} \geq a r_{n} / 2-a r_{n} / 16 \geq a r_{n} / 4
$$

which contradicts the fact that

$$
d_{\omega}(x, \gamma(i)) \leq d_{\omega}(x, y)+a r_{n} / 16 \leq \bar{d}(x, y)+a r_{n} / 8 \leq a r_{n} / 8+a r_{n} / 16<a r_{n} / 4 .
$$

This proves the claim.

By (3.1), the maximum of $\omega(e)$ over all edges on $\gamma$ is at most $b+D\left(r_{n} \log r_{n}\right)^{1 / 2} \leq D^{\prime}\left(r_{n} \log r_{n}\right)^{1 / 2}$ for some $D^{\prime}>0$. Therefore, one can find a vertex $z$ in $\gamma$ such that

$$
\left|\lambda d_{\omega}(x, y)-d_{\omega}(x, z)\right| \leq D^{\prime}\left(r_{n} \log r_{n}\right)^{1 / 2},
$$

and

$$
\left|(1-\lambda) d_{\omega}(x, y)-d_{\omega}(z, y)\right| \leq D^{\prime}\left(r_{n} \log r_{n}\right)^{1 / 2} .
$$

But then combining these inequalities with (3.1), we get

$$
|\lambda \bar{d}(x, y)-\bar{d}(x, z)| \leq 4 D^{\prime}\left(r_{n} \log r_{n}\right)^{1 / 2}
$$

and

$$
|(1-\lambda) \bar{d}(x, y)-\bar{d}(z, y)| \leq 4 D^{\prime}\left(r_{n} \log r_{n}\right)^{1 / 2},
$$

so that the proposition follows with $c=1 /\left(4 D^{\prime}\right)$.

\section{Proof of Proposition 1.6}

The proof of (1.6) only relies on the assumption that $N$ is increasing and unbounded. We assume that $r \geq 1$ is large enough so that $N(r) \geq 1$. Let $y \in B\left(x,\left(1+\frac{1}{N(r)}\right) r\right)$. Applying $\operatorname{SAG}^{*}(N)$ with $\lambda=1-2 / N(r)$ yields some $z \in X$ such that

$$
d(x, z) \leq\left(1-\frac{1}{N(r)}\right) d(x, y) \leq\left(1-\frac{1}{N(r)}\right)\left(1+\frac{1}{N(r)}\right) r \leq r
$$


and

$$
d(y, z) \leq \frac{3 d(x, y)}{N(r)} \leq \frac{6 r}{N(r)} .
$$

Hence (1.6) follows.

Let us turn to the proof of (1.5). There, we assume that $N$ satisfies the condition of Proposition 1.6. The following proposition implies (1.5) for the function $N / C^{\prime}$ and $\alpha_{0}=\beta$. Indeed, (1.5) corresponds to the case of a uniform subdivision of $[0,1]$, where $\lambda_{i}=i / m$, in which case $\alpha=\alpha_{i}=d(x, y) / m$.

Proposition 4.1. Assuming that $X$ is $\operatorname{SAG}^{*}(N)$, there exists $\beta \geq 0$ and $C^{\prime}$ such that for every sequence $\lambda_{0}=0<$ $\lambda_{1}<\cdots<\lambda_{m}=1$ and for all $x, y \in X$ such that $\alpha:=\min _{0 \leq i<m}\left(\lambda_{i+1}-\lambda_{i}\right) d(x, y) \geq \beta$, there exists a sequence $x=x_{0} \ldots, x_{m}=y$ satisfying, for all $0 \leq i \leq m-1$,

$$
d\left(x_{i}, x_{i+1}\right) \leq\left(1+\frac{C^{\prime}}{N(\alpha)}\right) \alpha_{i},
$$

where $\alpha_{i}=\left(\lambda_{i+1}-\lambda_{i}\right) d(x, y)$.

We start by proving a stronger version of (1.5) where $m=2^{k}$.

Lemma 4.2. Assuming that $X$ is $\mathrm{SAG}^{*}(N)$, there exists $\beta \geq 0$, and $C$ such that for all integer $q \geq 1$, and for all $x, y \in X$ such that $d(x, y) / 2^{q} \geq \beta$, there exists a sequence $x=x_{0} \ldots, x_{2^{q}}=y$ satisfying, for all $0 \leq i \leq$ $2^{q}-1$

$$
\frac{d(x, y)}{2^{q}}\left(1-\frac{C}{N\left(d(x, y) / 2^{q}\right)}\right) \leq d\left(x_{i}, x_{i+1}\right) \leq \frac{d(x, y)}{2^{q}}\left(1+\frac{C}{N\left(d(x, y) / 2^{q}\right)}\right) .
$$

Proof. On rescaling the metric by some number in $[1 / 2,1]$, we can assume without loss of generality that $d(x, y)=2^{n}$ for some $n \in \mathbb{N}$. We let $r_{0}=r_{0}^{\prime}:=d(x, y)$. Assuming that $n$ is large enough so that $2^{n} \geq \alpha_{0}$, where $\alpha_{0}$ is the parameter appearing in the definition $\mathrm{SAG}^{*}$, there exists $z$ such that ${ }^{2}$

$$
\min \{d(x, z), d(z, y)\} \geq \frac{r_{0}}{2}\left(1-\frac{1}{N\left(r_{0}\right)}\right)
$$

and

$$
\max \{d(x, z), d(z, y)\} \leq \frac{r_{0}}{2}\left(1+\frac{1}{N\left(r_{0}\right)}\right),
$$

for some constant $C$. We let $r_{1}=\max \{d(x, z), d(z, y)\}$ and $r_{1}^{\prime}=\min \{d(x, z), d(z, y)\}$ and apply SAG* to $(x, z)$ and $(z, y)$. Continuing this subdivision process as long as $r_{k-1}^{\prime} \geq \alpha_{0}$, and using that $r \rightarrow r(1+1 / N(r))$ is non-decreasing, we find a sequence $r_{1}, \ldots, r_{k}, \ldots$, satisfying

$$
r_{k} \leq \frac{r_{k-1}}{2}\left(1+\frac{1}{N\left(r_{k-1}\right)}\right)
$$

and

$$
r_{k}^{\prime} \geq \frac{r_{k-1}^{\prime}}{2}\left(1-\frac{1}{N\left(r_{k-1}^{\prime}\right)}\right)
$$

${ }^{2}$ Strictly speaking $\frac{1}{N\left(r_{0}\right)}$ should be $\frac{2}{N\left(r_{0}\right)}$ but this additional factor 2 will be absorbed in the constant $C$, so we prefer to get rid of it. 
and a sequence of finite sequences of vertices $x=z_{0}(k), \ldots, z_{2^{k}}(k)=y$ such that

$$
r_{k}^{\prime} \leq d\left(z_{i}(k), z_{i+1}(k)\right) \leq r_{k},
$$

for all $0 \leq i<2^{k}-1$.

Claim 4.3. There exists $n_{0} \geq 0$ such that for all $k \leq n-n_{0}, r_{k}^{\prime} \geq \alpha_{0}$, and

$$
\alpha_{0} \leq 2^{-k-1} r_{0} \leq r_{k}^{\prime} \leq r_{k} \leq 2^{-k+1} r_{0} .
$$

Proof. Note that the condition on $N$ implies that there exists $c>0$ such that for $r \geq \alpha_{0}, N(r) \geq c r^{\varepsilon}$. On enlarging $\alpha_{0}$ and reducing $\varepsilon$, we can assume that ${ }^{3} c=1$ and $\varepsilon \leq 1$.

Let $k \geq 2$, and observe that (4.1) and (4.2) respectively imply

$$
r_{k} \leq \frac{r_{k-1}}{2}\left(1+r_{k-1}^{-\varepsilon}\right)
$$

and

$$
r_{k}^{\prime} \geq \frac{r_{k-1}^{\prime}}{2}\left(1-r_{k-1}^{\prime-\varepsilon}\right)
$$

We do the following change of variable: $A_{k}=2^{k-n} r_{k}$ and $B_{k}=2^{k-n} r_{k}^{\prime}$. Note that $B_{0}=A_{0}=1$. We deduce from (4.3) and (4.4) that

$$
A_{k} \leq A_{k-1}+2^{\varepsilon(k-n)} A_{k-1}^{1-\varepsilon},
$$

and

$$
B_{k} \geq B_{k-1}-2^{\varepsilon(k-n)} B_{k-1}^{1-\varepsilon} \geq B_{k-1}-2^{\varepsilon(k-n)} A_{k-1}^{1-\varepsilon} .
$$

We will prove by induction on $k$ that

$$
1-2^{\frac{\varepsilon}{2}(k-n)} \leq B_{k} \leq A_{k} \leq 1+2^{\frac{\varepsilon}{2}(k-n)}
$$

as long as $k \leq n-n_{0}$, where ${ }^{4}$

$$
n_{0}=\max \left\{\lceil 2 / \varepsilon\rceil,\left\lceil\log _{2} \alpha_{0}\right\rceil+1, n_{1}\right\},
$$

where $n_{1}$ is the smallest integer such that $2^{-\frac{\varepsilon}{2}}+2^{\frac{-\varepsilon n_{1}}{2}+1} \leq 1$. This will prove the claim as this choice of $n_{0}$ implies

$$
\frac{1}{2} \leq B_{k} \leq A_{k} \leq 2,
$$

and $r_{k}^{\prime} \geq 2^{n_{0}-1} \geq \alpha_{0}\left(\right.$ since $\left.n_{0} \geq \log _{2} \alpha_{0}+1\right)$.

Let $1 \leq k \leq n-n_{0}$, and assume that (4.7) is satisfied for $k-1$. Since $n_{0} \geq 2 / \varepsilon$, we have that $B_{k-1} \geq 1 / 2$. Then, using that $n_{0} \geq \log _{2} \alpha_{0}$, we have that $r_{k-1}^{\prime} \geq 2^{n_{0}} \geq \alpha_{0}$. Therefore, $r_{k}$ and $r_{k}^{\prime}$ are both well-defined. Then, using (4.5) and the fact that $A_{k-1} \leq 2$, we obtain

$$
A_{k} \leq 1+2^{\frac{\varepsilon}{2}(k-n-1)}+2^{\varepsilon(k-n)+1} .
$$

Hence,

$$
2^{\frac{\varepsilon}{2}(n-k)}\left(A_{k}-1\right) \leq 2^{-\frac{\varepsilon}{2}}+2^{\frac{\varepsilon}{2}(k-n)+1} \leq 2^{-\frac{\varepsilon}{2}}+2^{\frac{-\varepsilon n_{0}}{2}+1} \leq 1,
$$

\footnotetext{
${ }^{3}$ This is only to simplify notation.

${ }^{4}$ We take $n$ to be large enough so that $n>n_{0}$.
} 
which proves the right-hand inequality of (4.7). Then, by (4.6), we have

$$
B_{k} \geq 1-2^{\frac{\varepsilon}{2}(k-n-1)}-2^{\varepsilon(k-n)+1},
$$

and we conclude similarly that

$$
2^{\frac{\varepsilon}{2}(n-k)}\left(1-B_{k}\right) \leq 1,
$$

proving the left-hand inequality of (4.7) and therefore the claim.

In what follows, we assume that $k \leq n-n_{0}$. By (4.1), (4.2) and the claim, we have

$$
r_{k} \leq \frac{r_{k-1}}{2}+\frac{2^{n-k+1}}{N\left(2^{n-k}\right)},
$$

and

$$
r_{k}^{\prime} \geq \frac{r_{k-1}^{\prime}}{2}-\frac{2^{n-k+1}}{N\left(2^{n-k}\right)}
$$

iterating these inequalities and using the property of $N$, one gets

$$
\begin{aligned}
r_{k} & \leq 2^{n-k}+\sum_{j=1}^{k} \frac{2^{n-k+1}}{N\left(2^{n-k+j-1}\right)} \\
& \leq 2^{n-k}+\frac{2^{n-k}}{N\left(2^{n-k}\right)} \sum_{j=1}^{k} \frac{2^{-\varepsilon(j-1)+1}}{\delta},
\end{aligned}
$$

hence writing $C=\sum_{j=1}^{\infty} \frac{2^{-\varepsilon(j-1)+1}}{\delta}$, we obtain

$$
r_{k} \leq 2^{n-k}+C \frac{2^{n-k}}{N\left(2^{n-k}\right)}
$$

and similarly,

$$
r_{k}^{\prime} \geq 2^{n-k}-C \frac{2^{n-k}}{N\left(2^{n-k}\right)} .
$$

Assuming that $k \geq n_{0}$, if we let $x=x_{0}=z_{0}(k), \ldots, x_{2^{k}}=z_{2^{k}}(k)=y$, then we deduce from (4.8) and (4.9) that for every $0 \leq i \leq 2^{k}-1$,

$$
\left(1-\frac{C}{N\left(d(x, y) / 2^{k}\right)}\right) d(x, y) / 2^{k} \leq d\left(x_{i}, x_{i+1}\right) \leq\left(1+\frac{C}{N\left(d(x, y) / 2^{k}\right)}\right) d(x, y) / 2^{k} .
$$

Hence Lemma 4.2 follows (with $\beta=2^{n_{0}}$ ).

Before proving Proposition 4.1, we first state the following immediate consequence of the definition of strong $N$-asymptotic geodesicity.

Lemma 4.4. Assuming that $X$ is $\mathrm{SAG}^{*}(N)$, then for all $\lambda_{0} \in(0,1 / 2]$, all $\lambda \in\left[\lambda_{0}, 1-\lambda_{0}\right]$, and all $x, y \in X$ with $d(x, y) \geq \alpha_{0}$, there exists $z \in X$ such that

$$
\left(1-\frac{1}{\lambda_{0} N(r)}\right) \lambda r \leq d(x, z) \leq\left(1+\frac{1}{\lambda_{0} N(r)}\right) \lambda r
$$


and

$$
\left(1-\frac{1}{\lambda_{0} N(r)}\right)(1-\lambda) r \leq d(z, y) \leq\left(1+\frac{1}{\lambda_{0} N(r)}\right)(1-\lambda) r,
$$

where $r=d(x, y)$.

Proof of Proposition 4.1. We now show how to deduce Proposition 4.1 from Lemma 4.2. Choose $q$ such that $2^{-q+2} d(x, y) \leq \alpha \leq 2^{-q+3} d(x, y)$ and assume that a sequence $x_{0}=x, x_{1}, \ldots, x_{2} q=y$ as in Lemma 4.2 has been constructed. In particular, one has that for all $0 \leq i \leq j \leq 2^{q}$,

$$
d\left(x_{i}, x_{j}\right) \leq\left(1+\frac{C}{N\left(d(x, y) / 2^{q}\right)}\right)(j-i) d(x, y) / 2^{q} .
$$

Let $0=v_{0}<\mu_{1}<v_{1}<\mu_{2}<v_{2}<\cdots<v_{m-1}<\mu_{m}=1$ be an increasing sequence of elements of $2^{-q} \mathbb{N}$ such that for every $1 \leq i \leq m-1, \lambda_{i}$ is a convex combination $\left(1-t_{i}\right) v_{i}+t_{i} \mu_{i}$, with $1 / 3 \leq t_{i} \leq 2 / 3$ (it is easy to see that this can be done). We let $z_{0}=x$ and $z_{m}=y$. For every $1 \leq i \leq m-1$, we apply Lemma 4.4 with $x=x_{2 q} \mu_{i}, y=x_{2 q} v_{i}$ and $\lambda=t_{i}$, yielding a constant $C_{1}$ and $z_{i} \in X$ such that

$$
d\left(x_{2 q} v_{i}, z_{i}\right) \leq\left(1+\frac{C_{1}}{N(\alpha)}\right) t_{i} d\left(x_{2 q} \mu_{i}, x_{2 q} v_{i}\right),
$$

and

$$
d\left(z_{i}, x_{2 q} \mu_{i}\right) \leq\left(1+\frac{C_{1}}{N(\alpha)}\right)\left(1-t_{i}\right) d\left(x_{2 q} \mu_{i}, x_{2 q} v_{i}\right)
$$

Three cases must be considered: the boundary values $d\left(z_{0}, z_{1}\right)$ and $d\left(z_{m-1}, z_{m}\right)$, and the generic case $d\left(z_{i}, z_{i+1}\right)$ for $1 \leq i \leq m-2$. We shall focus on the generic case, as the other two can be treated similarly.

We deduce from (4.10), from the choice of $\alpha$ (and the property of $N$ ) that there exists $C_{2}$ such that

$$
d\left(x_{2^{q}} \mu_{i}, x_{2^{q} v_{i}}\right) \leq\left(v_{i}-\mu_{i}\right) d(x, y)\left(1+\frac{C_{2}}{N(\alpha)}\right),
$$

and

$$
d\left(x_{2^{q} v_{i}}, x_{2^{q} \mu_{i+1}}\right) \leq\left(\mu_{i+1}-v_{i}\right) d(x, y)\left(1+\frac{C_{2}}{N(\alpha)}\right) .
$$

We deduce that there exists $C_{3}$ such that

$$
\begin{aligned}
& d\left(z_{i}, x_{2^{q} v_{i}}\right) \leq\left(v_{i}-\mu_{i}\right) t_{i} d(x, y)\left(1+\frac{C_{3}}{N(\alpha)}\right), \\
& d\left(x_{2^{q} \mu_{i+1}}, z_{i+1}\right) \leq\left(v_{i+1}-\mu_{i+1}\right)\left(1-t_{i+1}\right) d(x, y)\left(1+\frac{C_{3}}{N(\alpha)}\right) .
\end{aligned}
$$

By triangular inequality, we have

$$
d\left(z_{i}, z_{i+1}\right) \leq d\left(z_{i}, x_{2} q v_{i}\right)+d\left(x_{2 q} v_{i}, x_{2^{q}} \mu_{i+1}\right)+d\left(x_{2^{q}} \mu_{i+1}, z_{i+1}\right),
$$

which combined with the previous inequalities gives

$$
\begin{aligned}
d\left(z_{i}, z_{i+1}\right) & \leq\left(\left(v_{i}-\mu_{i}\right) t_{i}+\mu_{i+1}-v_{i}+\left(v_{i+1}-\mu_{i+1}\right)\left(1-t_{i+1}\right)\right) d(x, y)\left(1+\frac{C^{\prime}}{N(\alpha)}\right) \\
& =\left(\lambda_{i+1}-\lambda_{i}\right) d(x, y)\left(1+\frac{C^{\prime}}{N(\alpha)}\right)
\end{aligned}
$$


where $C^{\prime}=\max \left\{C_{2}, C_{3}\right\}$. Hence the sequence $x=z_{0}, z_{1}, \ldots, z_{m-1}, z_{m}=y$ satisfies the conclusion of Proposition 4.1.

\section{Strong asymptotical geodesicity implies limit shape}

This section is dedicated to the proof of "(i) implies (ii)" in Proposition 1.8.

\subsection{Notation and conventions}

Given a subset $A$ of $\mathbb{R}^{d}$, and $t \in \mathbb{R}$, we denote $t A=\{t a, a \in A\}$, and $A B=\{a+b, a \in A, b \in B\}$. So in particular $A^{n}=\left\{a_{1}+\cdots+a_{n}, a_{i} \in A\right\}$. We fix a norm $\|\cdot\|_{0}$ on $\mathbb{R}^{d}$. In the sequel, we consider the Hausdorff distance with respect to this norm: i.e. the Hausdorff distance between two compact subsets $A$ and $A^{\prime}$ of $\mathbb{R}^{n}$ is defined as

$$
d_{H}\left(A, A^{\prime}\right)=\sup \left\{r>0, A \subset A^{\prime} B_{\|\cdot\|_{0}}(0, r), A^{\prime} \subset A B_{\|\cdot\|_{0}}(0, r)\right\} .
$$

Observe that $A B_{\|\cdot\|_{0}}(0, r)$ is the set of points of $\mathbb{R}^{d}$ lying at distance at most $r$ from some point of $A$.

For every $x \in \mathbb{Z}^{d}$, we shall denote $B(x, r)$ the ball of center $x$ and radius $r$ for the distance $\delta$. Denote $\hat{B}(0, r)$ the convex hull of $B(0, r)$.

Note that since $\delta$ is bi-Lipschitz equivalent to $\|\cdot\|_{0}$, (ii) is equivalent to the existence of a norm $\|\cdot\|$, and of $C>0$ and $n_{2}$ such that for all $n \geq n_{2}$,

$$
d_{H}\left(\frac{1}{n} B(0, n), B_{\|\cdot\|}(0,1)\right) \leq \frac{C}{\phi(n)} .
$$

Finally, on replacing the distance $\delta$ by $\delta / \alpha_{0}$, we can assume that the constant $\alpha_{0}$ appearing in the definition of $\mathrm{SAG}(N)$ equals 1 , which we shall do in the sequel.

\subsection{Preliminary lemmas}

In what follows, we suppose that (i) is satisfied. The following lemma is the only place where we actually use this assumption.

Lemma 5.1. There exists $C^{\prime}$ such that for all $M \in \mathbb{N}^{*}$ and all $r \geq M$,

$$
d_{H}\left(\frac{1}{r} B(0, r / M)^{M}, \frac{1}{r} B(0, r)\right) \leq \frac{C^{\prime}}{\phi(r / M)} .
$$

Proof. First, note that

$$
B(0, r / M)^{M} \subset B(0, r) \subset B(0,(1+\varepsilon) r / M)^{M},
$$

where

$$
\varepsilon=\frac{1}{N(r / M)} \leq \frac{1}{c \phi(r / M)} .
$$

The left inclusion simply follows ${ }^{5}$ from triangular inequality, while the right inclusion results from (1.5). Recall that in restriction to $\mathbb{Z}^{d},\|\cdot\|_{0}$ and $\delta$ are bi-Lipschitz equivalent, so there exists a constant $G>0$ such that $\|x-y\|_{0} \leq$

\footnotetext{
${ }^{5}$ We also use (as we will throughout) the invariance of $\delta$.
} 
$G \delta(x, y)$ for all $x, y \in \mathbb{Z}^{d}$. Then, using (1.6), we have

$$
\begin{aligned}
B(0,(1+\varepsilon) r / M)^{M} & \subset\left([B(0, r / M)]_{6 \varepsilon r / M}\right)^{M} \\
& \subset\left(B(0, r / M) B_{\|\cdot\|_{0}}(0,6 G \varepsilon r / M)\right)^{M} \\
& =(B(0, r / M))^{M} B_{\|\cdot\|_{0}}(0,6 G \varepsilon r),
\end{aligned}
$$

where the notation $[B(0, r / M)]_{6 \varepsilon r / M}$ stands for the $6 \varepsilon r / M$-neighborhood of the ball $B(0, r / M)$ for the metric $\delta$. Hence the lemma follows with $C^{\prime}=6 G / c$.

We let $L$ be an integer $\geq 2$ to be determined later. Let $k \in \mathbb{N}$ such that $L^{k} \leq r<L^{k+1}$.

Corollary 5.2. There exists $C^{\prime \prime}$ (depending on $L$ ) such that for all $r, m \in \mathbb{N}$ with $m \geq 1$ and $r \geq L$ (i.e. $k \geq 1$ ),

$$
d_{H}\left(\frac{1}{m r} B(0, r / L)^{m L}, \frac{1}{m r} B(0, m r)\right) \leq \frac{C^{\prime \prime}}{\phi\left(L^{k}\right)} .
$$

Proof. Applying Lemma 5.1 yields that the left-hand term is at most $\frac{C^{\prime}}{\phi\left(L^{k-1}\right)}$. Using that $\phi\left(L^{k}\right) \leq L \phi\left(L^{k-1}\right)$, we deduce the corollary with $C^{\prime \prime}=L C^{\prime}$.

We now proceed with a innocent-looking lemma, that nevertheless concentrates the main feature of $\mathbb{Z}^{d}$ that is needed for the proof.

Lemma 5.3. Let $K$ be a compact symmetric subset of $\mathbb{R}^{d}$, and let $\hat{K}$ be its convex hull. Then, for all $n \geq d$, we have

$$
\hat{K}^{n}=K^{n-d} \hat{K}^{d}
$$

In particular, for all $n \geq 1$,

$$
d_{H}\left(\widehat{K^{n}}, \hat{K}^{n}\right) \leq d_{H}\left(K^{n}, \hat{K}^{n}\right) \leq d \cdot d_{H}(K, \hat{K}) .
$$

Proof. One inclusion is clear, so let us prove the other one. Let $x \in \hat{K}^{n}$. By convexity, $\hat{K}^{n}=n \hat{K}$, so that there exists $y \in \hat{K}$ such that $x=n y$. Now $y$ can be written as a convex combination $y=t_{0} y_{0}+\cdots+t_{d} y_{d}$ of $d+1$ elements of $K$. Write $n t_{i}=n_{i}+s_{i}$, where $s_{i} \in[0,1)$, and $n_{i}=\left[n t_{i}\right]$. Observe that the integer $m:=\sum_{i} s_{i}=n-\sum_{i} n_{i}$ satisfies $m<d+1$. Since, $\frac{1}{m} \sum_{i} s_{i} y_{i}$ is a convex combination of the $y_{i}$, it belongs to $\hat{K}$. So we have $\sum_{i} s_{i} y_{i} \in m \hat{K}=\hat{K}^{m}$. On the other hand, since $\sum_{i} n_{i}=n-m$, the element $\sum_{i} n_{i} y_{i}$ belongs to $K^{n-m}$. We deduce that $\hat{K}^{n} \subset K^{n-m} \hat{K}^{m} \subset$ $K^{n-d} \hat{K}^{d}$. To deduce the second inequality, we let $t=d_{H}(K, \hat{K})$ so that $\hat{K} \subset K B_{\|\cdot\|_{0}}(0, t)$. It follows (since $\mathbb{R}^{d}$ is abelian) that

$$
\hat{K}^{n} \subset K^{n-d} \hat{K}^{d} \subset K^{n-d} K^{d} B_{\|\cdot\|_{0}}(0, d t),
$$

so that $d_{H}\left(K^{n}, \hat{K}^{n}\right) \leq d t$.

Applying the second half of Lemma 5.3 with $K=B(0, r / L)$, and $n=L m$, we deduce following corollary. ${ }^{6}$

Corollary 5.4. For all $L>1$, and $r, m \in \mathbb{N}$ with $m \geq 1$,

$$
d_{H}\left(\frac{1}{m r} B(0, r / L)^{L m}, \frac{1}{m r} \hat{B}(0, r / L)^{L m}\right) \leq d \cdot d_{H}\left(\frac{1}{r} B(0, r / L), \frac{1}{r} \hat{B}(0, r / L)\right) .
$$

\footnotetext{
${ }^{6}$ In the right-hand side, we could have $\frac{1}{m r}$ instead of $\frac{1}{r}$, however the latter is enough for our purposes.
} 
On the other hand, combining Corollary 5.2 and Lemma 5.3, we obtain:

Corollary 5.5. For all $L>1$, and $r, m \in \mathbb{N}$ with $m \geq 1$ and $r \geq L$,

$$
d_{H}\left(\frac{1}{m r} \hat{B}(0, r / L)^{m L}, \frac{1}{m r} \hat{B}(0, m r)\right) \leq \frac{C^{\prime \prime}}{\phi\left(L^{k}\right)}+d \cdot d_{H}\left(\frac{1}{r} B(0, r / L), \frac{1}{r} \hat{B}(0, r / L)\right) .
$$

Proof. By Lemma 5.3, denoting $V$ for the convex hull of $B(0, r / L)^{m L}$, we have

$$
d_{H}\left(\frac{1}{r} V, \frac{1}{r} \hat{B}(0, r / L)^{m L}\right) \leq d \cdot d_{H}\left(\frac{1}{r} B(0, r / L), \frac{1}{r} \hat{B}(0, r / L)\right) .
$$

On the other hand for every pair $A, A^{\prime}$ of compact subsets of $\mathbb{R}^{n}$, we have that $d_{H}\left(\hat{A}, \hat{A}^{\prime}\right) \leq d_{H}\left(A, A^{\prime}\right)$. Hence taking the convex hull in Corollary 5.2 yields

$$
d_{H}\left(\frac{1}{m r} V, \frac{1}{m r} \hat{B}(0, m r)\right) \leq \frac{C^{\prime \prime}}{\phi\left(L^{k}\right)} .
$$

These two inequalities now give the corollary.

\subsection{Proof of (i) implies (ii) in Proposition 1.8}

We let $L>1$ to be determined later. We will prove by induction on $k$ the following Cauchy criterion for the sequence $\frac{1}{r} B(0, r)$ : there exists $C$ such that for all $k$, all $r \in \mathbb{N}$ such that $r \geq L^{k}$ and all positive integer $m$,

$$
d_{H}\left(\frac{1}{r} B(0, r), \frac{1}{m r} \hat{B}(0, m r)\right) \leq \frac{C}{\phi\left(L^{k}\right)} .
$$

Note that by triangular inequality, this implies that for all $t \in \mathbf{Q}$ such that $t \geq 1$,

$$
d_{H}\left(\frac{1}{r} B(0, r), \frac{1}{t r} B(0, t r)\right) \leq \frac{2 C}{\phi\left(L^{k}\right)},
$$

but since $\bar{d}$ takes values in a discrete set, we deduce that for all $r^{\prime}, r^{\prime \prime} \geq r$,

$$
d_{H}\left(\frac{1}{r^{\prime}} B\left(0, r^{\prime}\right), \frac{1}{r^{\prime \prime}} B\left(0, r^{\prime \prime}\right)\right) \leq \frac{4 C}{\phi\left(L^{k}\right)} .
$$

Observe that this both implies a Cauchy criterion for $\frac{1}{r} B(0, r)$ and the fact that the limit is a convex body (as it is also the limit of the sequence of convex bodies $\left.\frac{1}{r} \hat{B}(0, r)\right)$. Since $\phi$ is doubling, it also gives the right rate of convergence.

Note that since $\delta$ is bilipschitz equivalent to $\|\cdot\|_{0}$, there exists $r_{0}$ such that for all $r \geq 1$,

$$
\frac{1}{r} B(0, r) \subset B_{\|\cdot\|_{0}}\left(0, r_{0}\right) \text {. }
$$

So in particular,

$$
C_{0}:=\sup _{1 \leq r \leq L ; r \leq r^{\prime}} d_{H}\left(\frac{1}{r} B(0, r), \frac{1}{r^{\prime}} \hat{B}\left(0, r^{\prime}\right)\right)<\infty .
$$

Recall that $\eta: \mathbb{R}_{+} \rightarrow \mathbb{R}_{+}$is a function that satisfies $\lim _{\lambda \rightarrow \infty} \eta(\lambda) / \lambda=0$ and for all $\lambda>0, \phi(\lambda r) \leq \eta(\lambda) \phi(r)$. We can then choose $L$ such that

$$
\frac{d \eta(L)}{L} \leq 1 / 4
$$

and we let $C=\max \left\{C_{0} \phi(1), 4 C^{\prime \prime}\right\}$, where $C^{\prime \prime}$ is the constant of Corollary 5.2. 
Initial step: Since $C \geq C_{0} \phi(1)$, we have that (5.1) holds for $k=0$.

Induction hypothesis: We let $k \geq 1$, we assume that (5.1) holds for all $k^{\prime}<k$, all $L^{k^{\prime}} \leq r^{\prime}<L^{k^{\prime}+1}$ and all $m \geq 1$, and we let $r \in \mathbb{N}$ such that $r \geq L^{k}$.

We have, by triangular inequality,

$$
\begin{aligned}
d_{H}\left(\frac{1}{r} B(0, r), \frac{1}{m r} \hat{B}(0, m r)\right) \leq & d_{H}\left(\frac{1}{r} B(0, r), \frac{1}{r} B(0, r / L)^{L}\right) \\
& +d_{H}\left(\frac{1}{r} B(0, r / L)^{L}, \frac{1}{r} \hat{B}(0, r / L)^{L}\right) \\
& +d_{H}\left(\frac{1}{r} \hat{B}(0, r / L)^{L}, \frac{1}{m r} \hat{B}(0, r / L)^{m L}\right) \\
& +d_{H}\left(\frac{1}{m r} \hat{B}(0, r / L)^{m L}, \frac{1}{m r} \hat{B}(0, m r)\right) .
\end{aligned}
$$

Note that the third term is zero. The first term is taken care of by Corollary 5.2 (with $m=1$ ):

$$
d_{H}\left(\frac{1}{r} B(0, r), \frac{1}{r} B(0, r / L)^{L}\right) \leq \frac{C^{\prime \prime}}{\phi\left(L^{k}\right)} \leq \frac{C}{4 \phi\left(L^{k}\right)} .
$$

To deal with the second term, we apply Corollary 5.4 and the induction hypothesis (with $m=1$ ) as follows:

$$
\begin{aligned}
d_{H}\left(\frac{1}{r} \hat{B}(0, r / L)^{L}, \frac{1}{r} B(0, r / L)^{L}\right) & \leq d \cdot d_{H}\left(\frac{1}{r} \hat{B}(0, r / L), \frac{1}{r} B(0, r / L)\right) \\
& \leq \frac{d}{L} d_{H}\left(\frac{1}{(r / L)} \hat{B}(0, r / L), \frac{1}{(r / L)} B(0, r / L)\right) \\
& \leq \frac{d C}{L \phi\left(L^{k-1}\right)} \\
& \leq \frac{d C \eta(L)}{L \phi\left(L^{k}\right)} \\
& \leq \frac{C}{4 \phi\left(L^{k}\right)} .
\end{aligned}
$$

To treat the fourth term, we apply Corollary 5.5 and once again the induction hypothesis:

$$
\begin{aligned}
d_{H}\left(\frac{1}{m r} \hat{B}(0, r / L)^{m L}, \frac{1}{m r} \hat{B}(0, m r)\right) & \leq d \cdot d_{H}\left(\frac{1}{r} \hat{B}(0, r / L), \frac{1}{r} B(0, r / L)\right)+\frac{C}{4 \phi\left(L^{k}\right)} \\
& \leq \frac{C}{2 \phi\left(L^{k}\right)} .
\end{aligned}
$$

Combining these three inequalities proves (5.1), i.e.

$$
d_{H}\left(\frac{1}{r} B(0, r), \frac{1}{m r} \hat{B}(0, m r)\right) \leq \frac{C}{\phi\left(L^{k}\right)},
$$

which ends the proof that (i) implies (ii).

\section{Limit shape implies strong asymptotical geodesicity}

The aim of this section is to prove that (ii) implies (i) in Proposition 1.8. The proof is rather straightforward, so we will only prove (1.5), leaving (1.6) to the reader. 
Observe that since $\delta$ is an invariant distance on $\mathbb{Z}^{d}$, (ii) is equivalent to the fact that there exists $C>0$ and $\alpha>0$, such that for all $x, y \in \mathbb{Z}^{d}$ such that $\delta(x, y) \geq \alpha$,

$$
\|x-y\|\left(1-\frac{C}{\phi(\delta(x, y))}\right) \leq \delta(x, y) \leq\|x-y\|\left(1+\frac{C}{\phi(\delta(x, y))}\right) .
$$

Since $\phi$ is doubling, and since for $\delta(x, y)$ large enough, $\|x-y\| / 2 \leq \delta(x, y) \leq 2\|x-y\|$, on changing the constant $C$, we have

$$
\|x-y\|\left(1-\frac{C}{\phi(\|x-y\|)}\right) \leq \delta(x, y) \leq\|x-y\|\left(1+\frac{C}{\phi(\|x-y\|)}\right) .
$$

Now, fix two elements $x, y \in \mathbb{Z}^{d}$ and consider the segment $[x, y]$ in $\mathbb{R}^{d}$. We let $m \in \mathbb{N}$ and consider $x=$ $z_{0}, \ldots, z_{m}=y$ such that for all $0 \leq i \leq m, z_{i}=x+i(y-x) / m$. The $z_{i}$ are not necessarily in $\mathbb{Z}^{d}$, so we pick for each $1 \leq i \leq m-1$, some $x_{i} \in \mathbb{Z}^{d}$ such that $\left\|x_{i}-z_{i}\right\| \leq K$, where $K=\sup _{z \in \mathbb{R}^{d}} \inf _{v \in \mathbb{Z}}\|v-z\|$. We now have a sequence $x_{0}=x, \ldots, x_{m}=y$ of points in $\mathbb{Z}^{d}$ such that

$$
\left\|z_{i}-z_{i+1}\right\|-2 K \leq\left\|x_{i}-x_{i+1}\right\| \leq\left\|z_{i}-z_{i+1}\right\|+2 K .
$$

Let us assume that $\alpha=\|y-x\| / m$ is large enough, so that

$$
\frac{2 K}{\left\|z_{i}-z_{i+1}\right\|}=\frac{2 K}{\alpha} \leq \frac{C}{\phi(\alpha)}
$$

This is possible thanks to the fact that $\lim _{r \rightarrow \infty} \phi(r) / r=0$. We deduce that

$$
\left(1-\frac{C}{\phi(\alpha)}\right)\left\|z_{i}-z_{i+1}\right\| \leq\left\|x_{i}-x_{i+1}\right\| \leq\left(1+\frac{C}{\phi(\alpha)}\right)\left\|z_{i}-z_{i+1}\right\| .
$$

Since $\phi$ is increasing, we deduce from (6.1) that

$$
\left(1-\frac{C}{\phi(\alpha)}\right)\|x-y\| \leq \delta(x, y) \leq\left(1+\frac{C}{\phi(\alpha)}\right)\|x-y\|,
$$

and

$$
\left(1-\frac{C}{\phi(\alpha)}\right)\left\|x_{i}-x_{i+1}\right\| \leq \delta\left(x_{i}, x_{i+1}\right) \leq\left(1+\frac{C}{\phi(\alpha)}\right)\left\|x_{i}-x_{i+1}\right\| .
$$

Combining these two inequalities with (6.2), and assuming $\alpha$ large enough so that $C / \phi(\alpha) \leq 1 / 2$, we deduce

$$
\left(1-\frac{C}{\phi(\alpha)}\right)^{2}\left(1+\frac{C}{\phi(\alpha)}\right)^{-1} \delta(x, y) / m \leq \delta\left(x_{i}, x_{i+1}\right) \leq\left(1+\frac{C}{\phi(\alpha)}\right)^{2}\left(1-\frac{C}{\phi(\alpha)}\right)^{-1} \delta(x, y) / m .
$$

Hence,

$$
\left(1-4 \frac{C}{\phi(\alpha)}\right) \delta(x, y) / m \leq \delta\left(x_{i}, x_{i+1}\right) \leq\left(1+5 \frac{C}{\phi(\alpha)}\right) \delta(x, y) / m,
$$

so (1.5) follows.

\section{Appendix: Lower bound on $\bar{d}$}

In this section, we prove that a mild assumption on $v$ implies $\left(A_{2}\right)$. 
Lemma A.1. Let $X=(V, E)$ be a graph of degree $\leq q$. Assume that $v$ is supported on $[a, \infty)$ and that $v(\{a\})<$ $1 /(q-1)$. Then there exists $a^{\prime}>a$ and $r_{0}$ such that $\bar{d}(x, y) \geq a^{\prime} d(x, y)$ for all $x, y \in V$ such that $d(x, y) \geq r_{0}$.

Proof. For simplicity, let us assume that $a=0$. The assumption implies that there exists $\delta>0$ such that $\lambda:=v([0,0+$ $\delta])<1 /(q-1)$. Let $\varepsilon>0$ to be determined later. Let $\gamma$ be a path of length $n$ between two vertices $x$ and $y$. Assume that $\ell_{\omega}(\gamma) \leq \varepsilon d(x, y)$ and let $N$ be the number of edges of $\gamma$ whose $\omega$-lengths are $\geq \delta d(x, y)$. It follows that

$$
\delta N \leq \varepsilon n,
$$

so we deduce that $N \leq \varepsilon n / \delta$. This imposes that at least $(1-\varepsilon / \delta) n$ edges of $\gamma$ have $\omega$-length $\leq \delta$. Recall that by Stirling's formula, given some $0<\alpha<1$, the number of ways to choose $\alpha n$ edges in a path of length $\mathrm{n}$ is

$$
\sim \frac{n^{n}}{(\alpha n)^{\alpha n}((1-\alpha) n)^{(1-\alpha) n}}=(1 / \alpha)^{\alpha n}\left(1 /(1-\alpha)^{(1-\alpha) n} .\right.
$$

Thus the probability that $\gamma$ has $\omega$-length at most $\varepsilon n$ is less than a universal constant times

$$
\frac{\lambda^{(1-\varepsilon / \delta) n}}{(\varepsilon / \delta)^{(\varepsilon / \delta) n}(1-\varepsilon / \delta)^{(1-\varepsilon / \delta) n}}=\left(\frac{\lambda^{1-\varepsilon / \delta}}{(\varepsilon / \delta)^{\varepsilon / \delta}(1-\varepsilon / \delta)^{1-\varepsilon / \delta}}\right)^{n} .
$$

Note that

$$
\lim _{\varepsilon \rightarrow 0} \frac{\lambda^{1-\varepsilon / \delta}}{(\varepsilon / \delta)^{\varepsilon / \delta}(1-\varepsilon / \delta)^{1-\varepsilon / \delta}}=\lambda .
$$

We now pick $\varepsilon$ so that

$$
\lambda^{\prime}:=\frac{\lambda^{1-\varepsilon / \delta}}{(\varepsilon / \delta)^{\varepsilon / \delta}(1-\varepsilon / \delta)^{1-\varepsilon / \delta}} \leq \frac{1}{2}\left(\lambda+\frac{1}{q-1}\right) .
$$

On the other hand, the number of self-avoiding paths of length $\leq n$ is at most $q(q-1)^{n}$. We deduce that for this choice of $\varepsilon$, the probability that $d_{\omega}(x, y) \leq \varepsilon d(x, y)$ is at most a constant times $\sum_{n \geq d(x, y)}\left(\lambda^{\prime}(q-1)\right)^{n}$, which converges to 0 as $d(x, y) \rightarrow \infty$. Let $r_{0}$ be such that this probability is less than $1 / 2$ as soon as $d(x, y) \geq r_{0}$. It follows that for all $x, y \in V$ such that $d(x, y) \geq r_{0}$,

$$
\bar{d}(x, y) \geq \varepsilon d(x, y) / 2,
$$

which proves the lemma.

Corollary A.2. Let $X=(V, E)$ be a graph of degree $\leq q$. We assume that $\left(A_{1}\right)$ is satisfied, and that $v(\{0\})<1 / q$. Then there exists $a^{\prime \prime}>0$ such that $\bar{d}(x, y) \geq a^{\prime \prime} d(x, y)$ for all $x, y \in V$.

Proof. By the previous lemma, applied with $a=0$, there exists $r_{0}$, and some $a^{\prime}>0$ such that $\bar{d}(x, y) \geq a^{\prime} d(x, y)$ as soon as $d(x, y) \geq r_{0}$. On the other hand, the assumption implies that there exists $\delta>0$ such that $c:=v([0, \delta])<1$. Since the degree is at most $k$, the probability that the $\omega$-length of all vertices issued from a given vertex is at least $\delta$, is at least $(1-c)^{k}$. Hence the average distance between two distinct points is $\geq(1-c)^{k} \delta$. The corollary follows by taking $a^{\prime \prime}=\min \left\{a^{\prime},(1-c)^{k} \delta / r_{0}\right\}$.

\section{Acknowledgements}

I am grateful to Itai Benjamini for attracting my attention to this beautiful subject and for many inspiring discussions. I would like to thank Xuan Wang for suggesting a nice alternative notion of asymptotic geodesicity, and for his numerous remarks and corrections. Last but not least, I am indebted to both referees who read very carefully this manuscript, corrected some mistakes and helped improving its presentation. 


\section{References}

[1] D. Ahlberg. A Hsu-Robbins-Erdős strong law in first-passage percolation. Ann. Probab. 43 (2015) 1992-2025. MR3353820

[2] K. S. Alexander. Approximation of subadditive functions and convergence rates in limiting-shape results. Ann. Probab. 25 (1) (1997) $30-55$. MR1428498

[3] I. Benjamini and R. Tessera. First passage percolation on nilpotent Cayley graphs. Electron. J. Probab. 20 (2015) 1-20.

[4] E. Breuillard. Geometry of groups of polynomial growth and shape of large balls. Groups Geom. Dyn. 8 (3) (2014) 669-732.

[5] J. T. Cox and R. Durrett. Some limit theorems for percolation processes with necessary and sufficient conditions. Ann. Probab. 9(4) (1981) $583-603$.

[6] M. Damron and N. Kubota. Gaussian concentration for the lower tail in first-passage percolation under low moments. Available at arXiv:1406.3105.

[7] M. Damron N. Kubota. Rates of convergence in first passage percolation under low moment conditions.. Stochastic Process. Appl. 126 (10) (2016).

[8] G. Grimmett and H. Kesten. Percolation Since Saint-Flour. Percolation Theory at Saint-Flour, Probab. St.-Flour. Springer, Heidelberg, 2012. MR3014795

[9] H. Kesten. Aspects of first passage percolation. In École d'Été de probabilité de Saint-Flour XIV - 1984 125-264. Lecture Notes in Math. 1180. Springer, Berlin, 1986.

[10] H. Kesten. On the speed of convergence in first-passage percolation. Ann. Appl. Probab. 3 (2) (1993) 296-338.

[11] P. Pansu. Croissance des boules et des géodésiques fermées dans les nilvariétés. Ergodic Theory Dynam. Systems 3 (1983) $415-445$.

[12] D. Richardson. Random growth in a tessellation. Math. Proc. Cambridge Philos. Soc. 74 (1973) 515-528. MR0329079

[13] M. Talagrand. Concentration of measure and isoperimetric inequalities in product spaces. Publ. Math. Inst. Hautes Études Sci. 81(1 (1995) 73-205.

[14] R. Tessera. Volume of spheres in metric measured spaces and in groups of polynomial growth. Bull. Soc. Math. France 135 (1) (2007) 47-64.

[15] Y. Zhang. Shape fluctuations are different in different directions. Ann. Probab. 36 (1) (2008) 331-362.

[16] Y. Zhang. On the concentration and the convergence rate with a moment condition in first passage percolation. Stochastic Process. Appl. 120 (7) (2010) 1317-1341. MR2639748 